\title{
Salvage living-donor liver transplantation and ADV score
}

\author{
Shin Hwang, Gi-Won Song, Chul-Soo Ahn, Ki-Hun Kim, Deok-Bog Moon, Tae-Yong Ha, Dong-Hwan Jung, Gil-Chun Park, \\ Young-In Yoon, Sung-Gyu Lee
}

Department of Surgery, Asan Medical Center, University of Ulsan College of Medicine, Seoul, Korea

Background: Salvage liver transplantation is a definite treatment for recurrent hepatocellular carcinoma (HCC) after hepatectomy. ADV score is calculated by multiplying a-fetoprotein and des- $\gamma$-carboxyprothrombin concentrations and tumor volume. Prognostic accuracy of ADV score was assessed in patients undergoing salvage living-donor liver transplantation (LDLT) and their outcomes were compared with patients undergoing primary LDLT.

Methods: This study was retrospective single-center case-controlled study. Outcomes were compared in 125 patients undergoing salvage LDLT from 2007 to 2018 and in 500 propensity score-matched patients undergoing primary LDLT.

Results: In patients undergoing salvage LDLT, median intervals between hepatectomy and tumor recurrence, between first HCC diagnosis and salvage LDLT, and between hepatectomy and salvage LDLT were 12.0, 37.2, and 29.3 months, respectively. Disease-free survival (DFS, $P=0.98)$ and overall survival $(O S, P=0.44)$ rates did not differ significantly in patients undergoing salvage and primary LDLT. Pre-transplant and explant ADV scores were significantly predictive of DFS and OS in patients undergoing salvage and primary LDLT $(P<0.001)$. DFS after prior hepatectomy $(P=0.52)$ and interval between hepatectomy and $L D L T(P=0.82)$ did not affect DFS after salvage LDLT. Milan criteria and ADV score were independently prognostic of DFS and OS following salvage LDLT, and prognosis of patients within and beyond Milan criteria could be further stratified by ADV score.

Conclusions: The risk factors and post-transplant outcomes were similar in patients undergoing salvage and primary LDLT. ADV scores were shown to be an integrated surrogate biomarker for post-transplant prognosis in salvage and primary LDLT recipients. This prediction model using pre-transplant and explant ADV scores can therefore provide reliable information on the post-transplant prognosis, thus assisting in the decision whether to perform salvage LDLT. 